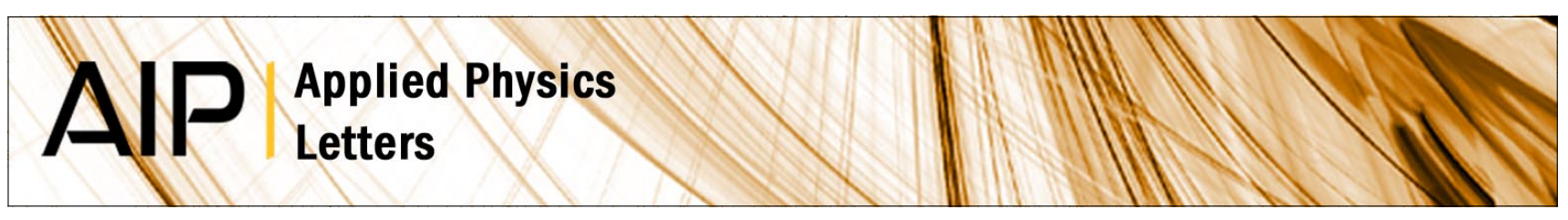

\title{
Tunable magneto-photonic response of nickel nanostructures
}

J. F. Torrado, J. B. González-Díaz, G. Armelles, A. García-Martín, A. Altube et al.

Citation: Appl. Phys. Lett. 99, 193109 (2011); doi: 10.1063/1.3660587

View online: http://dx.doi.org/10.1063/1.3660587

View Table of Contents: http://apl.aip.org/resource/1/APPLAB/v99/i19

Published by the American Institute of Physics.

Additional information on Appl. Phys. Lett.

Journal Homepage: http://apl.aip.org/

Journal Information: http://apl.aip.org/about/about_the_journal

Top downloads: http://apl.aip.org/features/most_downloaded

Information for Authors: http://apl.aip.org/authors

\section{ADVERTISEMENT}

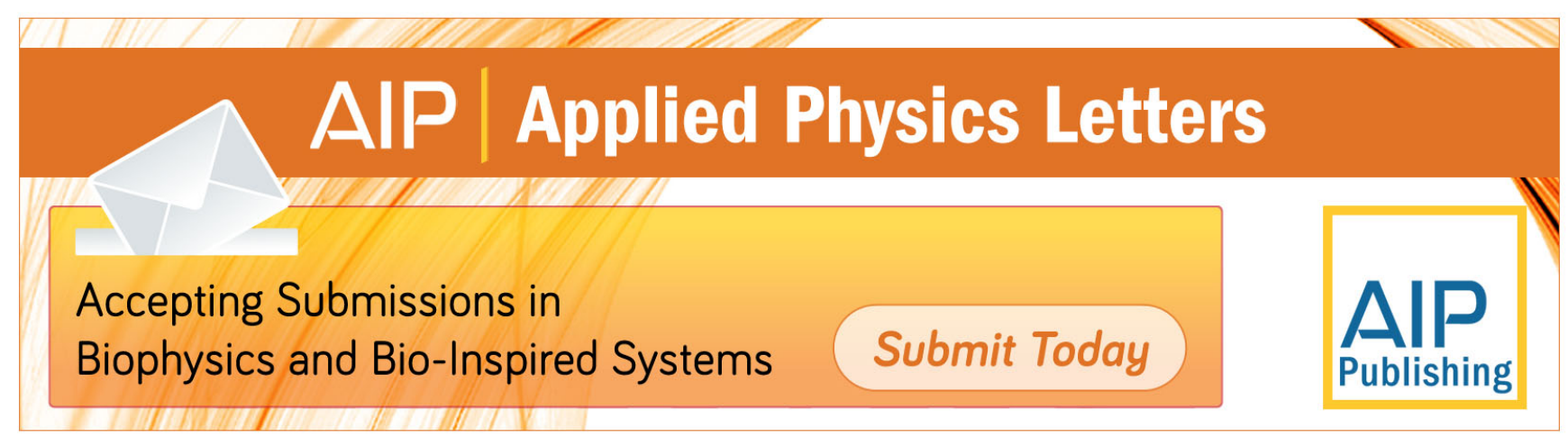




\title{
Tunable magneto-photonic response of nickel nanostructures
}

\author{
J. F. Torrado, ${ }^{1}$ J. B. González-Díaz, ${ }^{1, a)}$ G. Armelles, ${ }^{1}$ A. García-Martín, ${ }^{1, b)}$ A. Altube, ${ }^{2}$ \\ M. López-García, ${ }^{2}$ J. F. Galisteo-López, ${ }^{2}$ A. Blanco, ${ }^{2}$ and C. López ${ }^{2}$ \\ ${ }^{1}$ IMM-Instituto de Microelectrónica de Madrid (CNM-CSIC), C/Isaac newton 8, PTM, Tres Cantos, \\ 28760 Madrid, Spain \\ ${ }^{2}$ Instituto de Ciencia de Materiales de Madrid (CSIC) and Unidad Asociada CSIC-U. Vigo, C/Sor Juana Inés \\ de la Cruz 3, 28049 Madrid, Spain
}

(Received 8 August 2011; accepted 23 October 2011; published online 11 November 2011)

\begin{abstract}
In this letter, we present both experimental and numerical studies of the magneto-optical (MO) properties of nickel infiltrated opals. Ni can show interesting MO properties that can be controlled by nanostructuration through colloidal crystals templating. Nanostructuration allows the coupling of light to surface plasmon modes of $\mathrm{Ni}$, and a clear dependence of the $\mathrm{MO}$ response as a function of the structural parameters of the template has been observed. This dependence can be used in future tunable devices such as switchers or MO modulators. (C) 2011 American Institute of Physics. [doi:10.1063/1.3660587]
\end{abstract}

Photonic crystals are periodic dielectric materials that can control the flow of light in a very versatile way in one, two, or three dimensions (1D, 2D, or 3D) and for a large energy scale. Since their proposal, more than twenty years ago, ${ }^{1,2}$ most efforts centered on the fabrication and further optimization of systems using non-absorbing materials (dielectrics), those for which theoretical predictions were first developed. Recently, there have been new efforts to consider non-passive optical systems to actively control light propagation, where absorption or other non-linear phenomena play an important role. Magnetic photonic crystals (or magnetophotonic crystals, MPCs) are one of these new approaches. ${ }^{3,4}$ The main goal is to use magnetic fields to control the photonic properties of different nanostructures incorporating magneto-optical (MO) active materials In such a way, MPCs have been fabricated mainly in 1D, alternating magnetic and dielectric layers, enhancing MO Faraday and Kerr effects, or improving second harmonic generation, among others. ${ }^{5}$ In the case of $2 \mathrm{D}$ MPCs they have been prepared from porous alumina matrices ${ }^{6,7}$ or via nanosphere lithography. ${ }^{89}$ In 3D MPCs have been prepared from selfassembled colloidal structures ${ }^{10}$ by synthesizing ferrite within the artificial opal voids ${ }^{11}$ or by impregnating them with maghemite nanoparticles. ${ }^{12}$

Nanostructured metals (i.e., metallic PCs) have been profusely used in the framework of plasmonic nanostructures. In this regard nickel is a very promising candidate to explore MO effects in such photonic structures ${ }^{13}$ due to its versatile incorporation into different porous structures from a number of different techniques, such as electrochemistry, ${ }^{7}$ atomic layer deposition, ${ }^{14}$ or by simply direct nanoparticle infiltration. ${ }^{15}$

In this letter, we describe how properly nanostructured $\mathrm{Ni}$ exhibits remarkable MO responses, which can be controlled in a simple way by colloidal crystals templating. We have found that the MO response exhibits features that can

\footnotetext{
a) Present address: Nanomagnetism Group, CIC nanoGUNE Consolider, Tolosa Hiribidea, 76, E-20018 Donostia-San Sebastián, Spain.

b)Electronic mail: a.garcia.martin@csic.es.
}

be controlled by fine tuning of the structural parameters of the template. Such features are associated to the excitation of surface plasmon (SP) modes of Ni. The magnitude of the features is found also to depend on the long-range order of the sample.

Ordered arrays of monodisperse polystyrene (PS) spheres supported onto gold sputtered microscope slides were used as templates. PS artificial opals were grown by vertical deposition method, ${ }^{16}$ which provides highly ordered face centered cubic (fcc) structures with controlled thickness and orientation. Typical sample thickness used for metal deposition ranges from $100 \mathrm{~nm}$ to $4-5 \mu \mathrm{m}$. Ni was infiltrated potentiostatically at $\mathrm{E}=-1 \mathrm{~V}$ vs $\mathrm{Ag} / \mathrm{AgCl}$. Electrochemical deposition experiments were carried out using a conventional three-electrode electrochemical cell and controlled using an Autolab PGSTAT30 potentiostat-galvanostat. The active area was about $1 \mathrm{~cm}^{2}$ delimited by a mask of nail varnish. All experiments were performed at room temperature. Ni layer thickness, proportional to deposition time, was controlled to reach a half sphere. After infiltration, the original polymer template was dissolved in cyclohexane. Figure 1 shows experimental procedure scheme together with atomic force microscopy (AFM) images of three different templates, corresponding to sphere diameters of 320,457 , and $780 \mathrm{~nm}$. Although the sample crystalline ordering is always fair, postprocessing stage can somehow affect the final quality of the structure, as can be seen in Figure 1 for the 320 and $780 \mathrm{~nm}$ structures. This issue can appear after polymer removal.

Polar Kerr rotation (PKR) (magnetic field applied normal to the sample plane) and transversal MO Kerr effect (TMOKE) (magnetic field applied parallel to the sample and perpendicular to the incident light plane) spectra were measured in the visible range (spot size around $1 \mathrm{~mm}^{2}$ ) applying a magnetic field high enough to reach saturation $(1.2 \mathrm{~T}$ and 350 Gauss, respectively). The modeling of the MO response has been realized using a scattering matrix method (SMM) that is able to account for MO effects ${ }^{17}$ for perfect periodic structures.

Figure 2 summarizes PKR results (experimental and theoretical) for different sphere sizes. Whereas the spectra of 

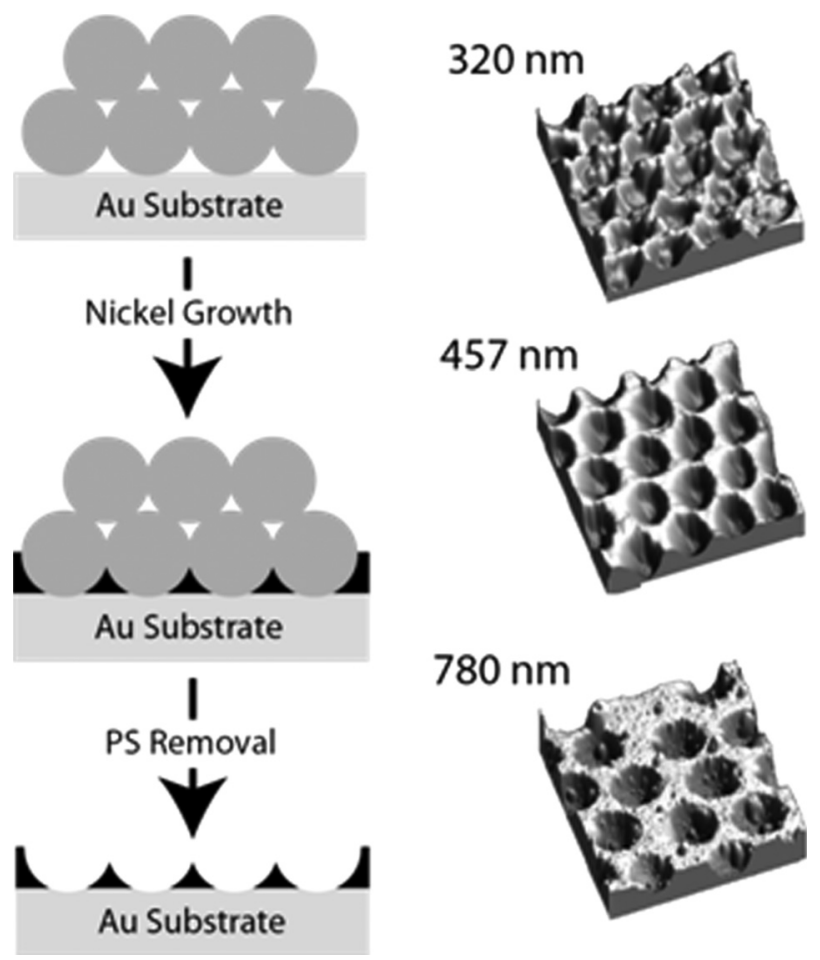

FIG. 1. Left: schematic representation of sample preparation. Right: AFM images different templates with increasing sphere size.

the 320 and $457 \mathrm{~nm}$ samples show only one feature in the near UV region, the spectra of the $780 \mathrm{~nm}$ sample have two clear features: one in the UV region and the other in the near infrared region. These features are due to SP modes of $\mathrm{Ni}$. Surface periodic corrugation offsets the momentum mismatch so that the in-plane wave vector of light matches that of surface plasmon polaritons (SPPs) modes of Ni. At normal incidence, the conservation of the in-plane momentum can be written as $k_{\mathrm{spp}}= \pm i b_{1} \pm j b_{2}$, with $b_{1}$ and $b_{2}$ the reciprocal lattice vector for the hexagonal lattice (see scheme in Figure 2)

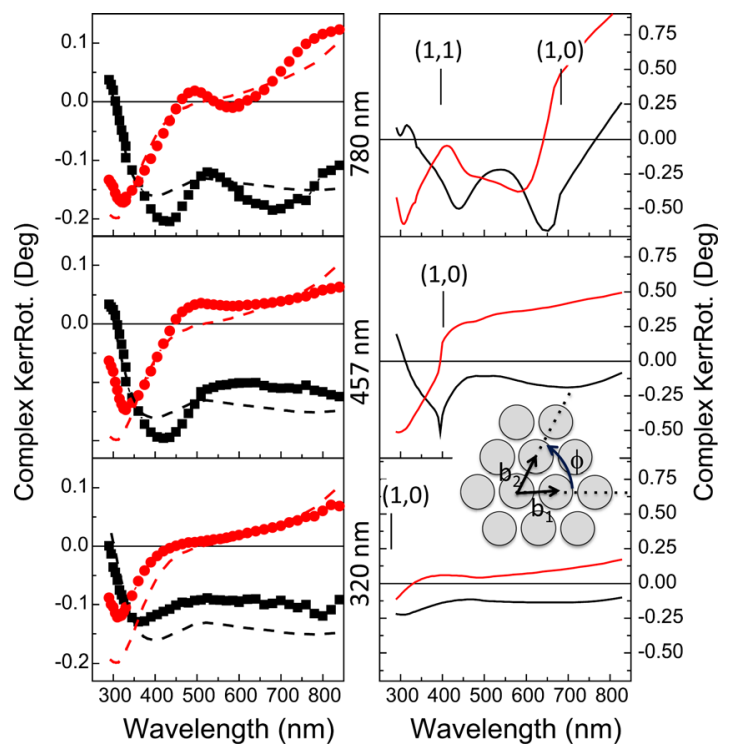

FIG. 2. (Color online) Experimental (dots, left) and theoretical (lines, right) results for Kerr rotation (in black) and ellipticity (in red): from top to bottom: $780 \mathrm{~nm}, 457 \mathrm{~nm}$, and $320 \mathrm{~nm}$. The dashed lines (left) correspond to bulk Ni. Vertical bars (right) mark frequencies of the folded SPP modes for a Ni/Air system. and $i$ and $j$ integers. The vertical bars in Figure 2 represent the frequencies of the SPP modes for a Ni/Air system and $i=1$ and $j=0$ (or $i=0$ and $j=1$ ) or $i=j=1$. As can be observed, the features are localized around such frequencies and follow the same sphere diameter dependence. Therefore, we associate the structures as arising from SP modes originated from the folding of the SPP dispersion curve. In the spectral region of the discussed features we observe an increase of the MO response with respect to bulk $\mathrm{Ni}$, but contrary to what have been observed in other nanostructured systems, ${ }^{7}$ such increase is not due to an enhancement of the polarization conversion coefficient, $r_{p s}$, but to a reduction of the reflectivity, $r{ }^{18}$ In fact $r_{\mathrm{ps}}$ shows a decrease of its intensity in the spectral region of these features, which suggests that the SP modes, arising from folding the SPP dispersion curve, interacts with the SP localized at the voids. Such coupling will reduce the intensity of the electromagnetic field inside $\mathrm{Ni}$ and, therefore, $r_{\mathrm{ps}}$. This coupling between propagating and localized modes has also been observed in similar Au nanostructures. ${ }^{19}$

Although the shape and sphere diameter dependence of experimental and theoretical spectra are very similar, the intensity of the experimental PKR is not as high as theoretically expected from SMM simulations (solid line in Figure 2), probably due to disorder that damps and broadens the resonances, where geometrical disorder affects the most. Disorder in these structures usually appears at long-range being less pronounced at short range. To verify this assumption, angle-resolved reflectance measurements were performed. By imaging the back focal plane of a high numerical aperture $(\mathrm{NA}=0.75)$ optical microscope objective under monochromatic illumination the optical Fourier transform of the sample reflectance was obtained. ${ }^{20}$ The reflectance patterns obtained in this manner are equifrequency surfaces (EFSs) of the 2D $\mathrm{PhC}^{21}$ where the dark streaks correspond to modes of the system to which one can couple to when impinging on the sample. Figure 3 shows experimental EFSs taken with 2 different spot sizes (50 and $10 \mu \mathrm{m}$ in Figures 3(a) and 3(b), respectively) centered at the same point of a $780 \mathrm{~nm}$ pitch sample illuminated with $633 \mathrm{~nm}$ light. Note the six-fold symmetry corresponding to the crystal order. Common defects in this kind of structures are rotated domains, where the sample consists of several single crystal regions which are slightly rotated with respect to each other. Hence, as one probes large sample regions comprising several domains, different features in the EFSs are expected to wash

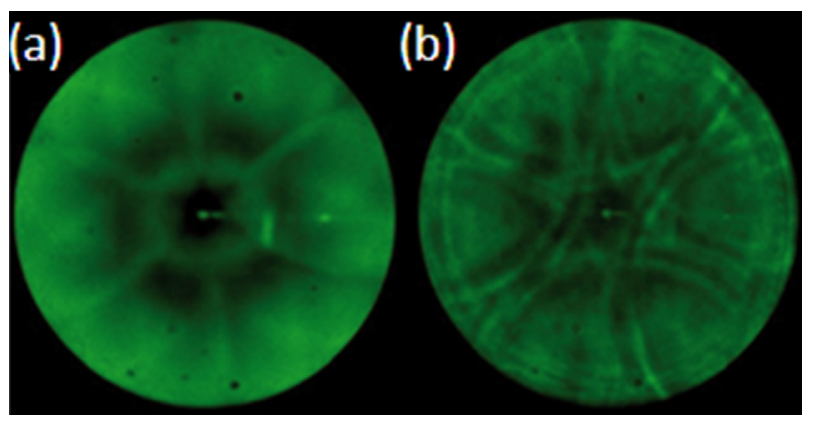

FIG. 3. (Color online) Angle-resolved reflectance of $780 \mathrm{~nm}$ Ni structure for two different spot sizes: (a) $50 \mu \mathrm{m}$ and (b) $10 \mu \mathrm{m}$. 


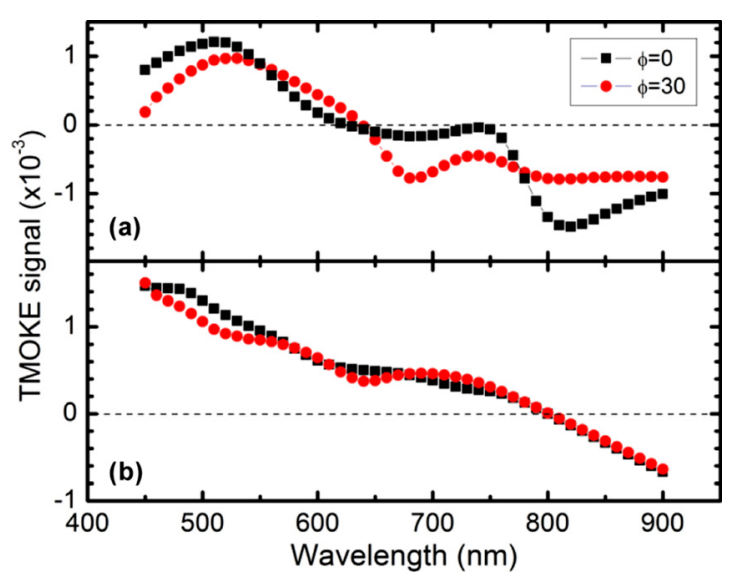

FIG. 4. (Color online) Experimental TMOKE spectra taken at $\theta=45^{\circ}$ for the $457 \mathrm{~nm}$ sample (a) and for the $780 \mathrm{~nm}$ sample (b), at two different inplane angles, $\phi$. In (a) a clear difference in the two measurements can be observed, reflecting the difference in the crystal orientation, whereas in (b) this difference is smeared out to a large extent, due to disorder effects.

out. This can be seen in Fig. 3(a) for the large spot size $(50 \mu \mathrm{m})$ measurements. For smaller spot sizes of $10 \mu \mathrm{m}$ (Figure 3(b)) different features are more clearly defined, a signature of a better crystallographic order. This is particularly relevant when different crystallographic directions are to be studied, like in TMOKE measurements, as discussed below.

TMOKE measures the difference in the reflectance when the direction of a magnetic field, applied in the sample plane and perpendicular to the incident beam, is reversed $\left(\mathrm{TMOKE}=\left(\mathrm{R}^{+}-\mathrm{R}^{-}\right) /\left(\mathrm{R}^{+}+\mathrm{R}^{-}\right)\right)$. The TMOKE signal can be obtained at different incident angle $\theta$ and for different azimuth angles $\phi$ in order to explore different crystallographic directions. $^{22}$ It must be noted that $\theta=0^{\circ}$ means incident light exactly at $\Gamma$ point and at this point the TMOKE signal is identically zero. However by increasing $\theta$ we scan the different high symmetry reciprocal space directions $\Gamma \mathrm{M}$ $\left(\phi=0^{\circ}\right)$ or $\Gamma \mathrm{K}\left(\phi=30^{\circ}\right)$. This procedure is routinely used in $2 \mathrm{D}$ photonic crystals optical characterization and allows retrieving band structures in standard reflection experiments (obtaining similar results to Figure 3(b)). For simplicity, here we will focus on incidence at $\theta=45^{\circ}$. Results for the $457 \mathrm{~nm}$ sample are shown in Figure 4(a) where a clear dependence on $\phi$ in the TMOKE signal can be observed, confirming relatively good crystalline order in a rather longrange. However, this dependence is almost completely smeared out in the $780 \mathrm{~nm}$ sample (Figure 4(b)). This is an indication of an increase in disorder, that can arise both from individual elements (the voids) and from lattice imperfections, which have a pronounced influence given the probe beam size used $(\sim 1 \mathrm{~mm})$, as previously discussed.

In conclusion, we have prepared MPCs from selfassembled polymeric monolayers replicated on nickel. These structures exhibit an increase of the magneto-optical response with features at spectral regions controllable through structural parameters of the template. These features are due to the excitation of Ni Surface Plamons modes. We have found that the effect of disorder on the PKR is to decrease the amount of that enhancement. In the case of TMOKE, since it is largely affected by the crystal orientation probed, the effect of long- range disorder significantly alters the signal.

We acknowledge J.M. García-Martín for the AFM images. This work has been partially supported by EU FP7 (NoE Nanophotonics4Energy Grant No. 248855 and NMP3SL-2008-214107-Nanomagma); the CSIC PIF08-016, the Spanish MICINN (CSD2007-0046-Nanolight.es, CSD200800023-Funcoat, MAT2009-07841-GLUSFA, MAT200806765-C02-01/NAN-MAGPLAS) and Comunidad de Madrid (S2009/MAT-1756-PHAMA and S2009/TIC-1476MICROSERES).

${ }^{1}$ S. John, Phys. Rev. Lett. 58, 2486 (1987).

${ }^{2}$ E. Yablonovitch, Phys. Rev. Lett. 58, 2059 (1987).

${ }^{3}$ M. Inoue, R. Fujikawa, A. Baryshev, A. Khanikaev, P. B. Lim, H. Uchida, O. Aktsipetrov, A. Fedyanin, T. Murzina, and A. Granovsky, J. Phys. D 39, R151 (2006).

${ }^{4}$ G. Armelles, A. Cebollada, A. Garcia-Martin, J. M. Garcia-Martin, M. U. Gonzalez, J. B. Gonzalez-Diaz, E. Ferreiro-Vila, and J. F. Torrado, J. Opt. A, Pure Appl. Opt. 11, 114023 (2009).

${ }^{5}$ A. A. Fedyanin, O. Aktsipetrov, D. Kobayashi, K. Nishimura, H. Uchida, and M. Inoue, IEEE Trans. Magn. 40, 2850 (2004).

${ }^{6}$ Y. Ikezawa, K. Nishimura, H. Uchida, and M. Inoue, J. Magn. Magn. Mater. 272, 1690 (2003).

7J. B. Gonzalez-Díaz, A. García-Martín, G. Armelles, D. Navas, M. Vázquez, K. Nielsch, R. B. Wehrspohn, and U. Gösele, Adv. Mater. 19, 2643 (2007).

${ }^{8}$ G. Ctistis, E. Papaioannou, P. Patoka, J. Gutek, P. Fumagalli, and M. Giersig, Nano Lett. 9, 1 (2009).

${ }^{9}$ E. T. Papaioannou, V. Kapaklis, P. Patoka, M. Giersig, P. Fumagalli, A. Garcia-Martin, E. Ferreiro-Vila, and G. Ctistis, Phys. Rev. B 81, 054424 (2010).

${ }^{10}$ J. F. Galisteo-López, M. Ibisate, R. Sapienza, L. Froufe, A. Blanco, and C. Lopez, Adv. Mater. 23, 30 (2011).

${ }^{11}$ K. Nishimura, T. Kodama, A. Baryshev, H. Uchida, and M. Inoue, J. Appl. Phys. 95, 6633 (2004).

${ }^{12}$ J. M. Caicedo, E. Taboada, D. Hrabovsky, M. Lopez-Garcia, G. Herranz, A. Roig, A. Blanco, C. Lopez, and J. Fontcuberta, J. Magn. Magn. Mater. 322, 1494 (2010).

${ }^{13}$ K. Napolskii, N. Sapoletova, A. Eliseev, G. Tsirlina, A. Rubacheva, E. Gan'shina, M. Kuznetsov, M. Ivanov, V. Valdner, E. Mishina, et al., J. Magn. Magn. Mater. 321, 833 (2009).

${ }^{14}$ X. D. Yu, Y.-J. Lee, R. Furstenberg, J. O. White, and P. V. Braun. Adv. Mater. 19, 1689 (2007).

${ }^{15}$ J. M. Caicedo, O. Pascu, M. Lopez-Garcia, V. Canalejas, A. Blanco, C. Lopez, J. Fontcuberta, A. Roig, and G. Herranz, ACS Nano 5, 2957 (2011).

${ }^{16}$ P. Jiang, J. F. Bertone, K. S. Hwang, and V. L. Colvin, Chem. Mater. 11, 2132 (1999).

${ }^{17}$ A. Garcia-Martin, G. Armelles, and S. Pereira, Phys. Rev. B 71, 205116 (2005).

${ }^{18}$ At normal incidence, the complex polar Kerr rotation, $\theta+\mathrm{i} \phi$, the reflectivity, $r$, and $r_{\mathrm{ps}}$ are related as $\theta+\mathrm{i} \phi=r_{\mathrm{ps}} / r$, with $\theta$ and $\phi$ the polar Kerr rotation and ellipticity, respectively. The enhancement can be due to an increase of $r_{\mathrm{ps}}$, a decrease of the reflectivity, $r$, or a combined effect.

${ }^{19}$ T. A. Kelf, Y. Sugawara, R. M. Cole, J. J. Baumberg, M. E. Abdelsalam, S. Cintra, S. Mahajan, A. E. Russell, and P. N. Bartlett, Phys. Rev. B 74, 245415 (2006).

${ }^{20}$ M. Richard, J. Kasprzak, R. Romestain, R. André, and L. S. Dang, Phys. Rev. Lett 94, 187401 (2005).

${ }^{21}$ M. Notomi, Phys. Rev. B 62, 10696 (2000).

${ }^{22}$ J. F. Torrado, E. T. Papaioannou, G. Ctistis, P. Patoka, M. Giersig, G. Armelles, and A. Garcia-Martin, Phys. Status Solidi (RRL) 4, 271 (2010). 